\title{
Myasthenia Associated with Other Autoimmune Diseases: A Series of Cases
}

\author{
Boundia Djiba, Nafissatou Diagne, Mohamed Dieng, Baidy Sy Kane, Atoumane Faye, \\ Awa Cheikh Ndao, Maimouna Sow, Abdoulaye Pouye \\ Department of Internal Medicine, Aristide Ledantec Hospital, Dakar, Senegal \\ Email: boundiadjiba@yahoo.fr
}

How to cite this paper: Djiba, B., Diagne, N., Dieng, M., Kane, B.S., Faye, A., Ndao, A.C., Sow, M. and Pouye, A. (2020) Myasthenia Associated with Other Autoimmune Diseases: A Series of Cases. Open Journal of Internal Medicine, 10, 44-50.

https://doi.org/10.4236/ojim.2020.101005

Received: December 3, 2019

Accepted: February 23, 2020

Published: February 26, 2020

Copyright $\odot 2020$ by author(s) and Scientific Research Publishing Inc. This work is licensed under the Creative Commons Attribution International License (CC BY 4.0).

http://creativecommons.org/licenses/by/4.0/

\begin{abstract}
We report four observations of myasthenia gravis associated with other autoimmune diseases. Myasthenia gravis can be associated with all autoimmune diseases with a predominance of dysthyroidism. Among the autoimmune diseases associated with myasthenia gravis in our series, there were associations with hyperthyroidism, sjogren syndrome, Biermer's disease. You would have to know how to look for another autoimmune disease in front of all myasthenia gravis by looking for the slightest sign of appeal that could point you towards another pathology.
\end{abstract}

\section{Keywords}

Myathenia Gravis, Autoimmun Disease, Association

\section{Introduction}

Myasthenia gravis is defined as an autoimmune disease affecting the neuromuscular junction. It can be associated with almost all autoimmune diseases. Its incidence is globally low, from 100 to 200 for 1 million inhabitants and its association with other autoimmune diseases is also rare [1]. The association with dysthyroidism is the most common with $5 \%$ to $10 \%$ of cases, however, other autoimmune diseases can be found [2]. We report 4 observations of myasthenia associated with other autoimmune diseases.

\section{Observations}

\section{Observation 1}

This was a 31-year-old female patient who was already being followed for megaloblastic anemia with B12 vitamin therapy prior to admission. She was received in February 2015 for an intense fatiguability at the least effort and the 
examination at the entrance found a palmar-plantar melanoderma associated with unilateral ptosis and difficulty to hold her arms on the horizontal plane for a period of 30 seconds. The prostigmine test resulted in a marked improvement in clinical symptomatology.

At the paraclinic, the hemogram showed a hemoglobin level of $12 \mathrm{~g} / \mathrm{dl}$, the platelet count was $369,000 / \mathrm{mm}^{3}$ and the leucocyte count was 8140 elements $/ \mathrm{mm}^{3}$. The ultra sensitive antithyrostimuline antibodies assay showed a level of 1.47 $\mathrm{mIU} / \mathrm{L}$ (normal between 0.27 and 4.2), the search for antibodies against thymic cell extracts (anti-ECT), antibodies against citrrulinated cyclic peptides (anti-CCP2) and anti-thyroid peroxydase antibodies (anti-TPO) was negative. The search for anti-parietal cell antibodies was negative and the search for antibodies against intrinsic factors was positive. The anti-musclespecific kinase

Autoantibody (anti-MUSK) assay was 0.03 (normal less than 0.05) and the assay for anti-acetylcholine receptor antibodies 2 times was less than 0.20 . Oeso-gastroduodenal fibroscopy found an appearance of atrophic fundic gastritis and histology favored intestinal metaplasia. The electroneuromyogram revealed an aspect compatible with myasthenia (Figure 1).

The thoracic CT was peculiar. The other explorations were without particularities

The diagnosis of seronegative myasthenia gravis associated with Biermer's disease was selected.

L'evolution est stable à ce jour avec une reprise acceptable des activités et une survenue de facon intermittente et rare de crises sous azathioprine $100 \mathrm{mg} / \mathrm{day}$ associé a la vitamine B12, une dose tous les mois.

\section{Observation 2}

A 32-year-old female patient with a history of total thyroidectomy in 1996 for goitre (under levothyroxine sodium) and acute polyradiculoneuropathy in 2012,

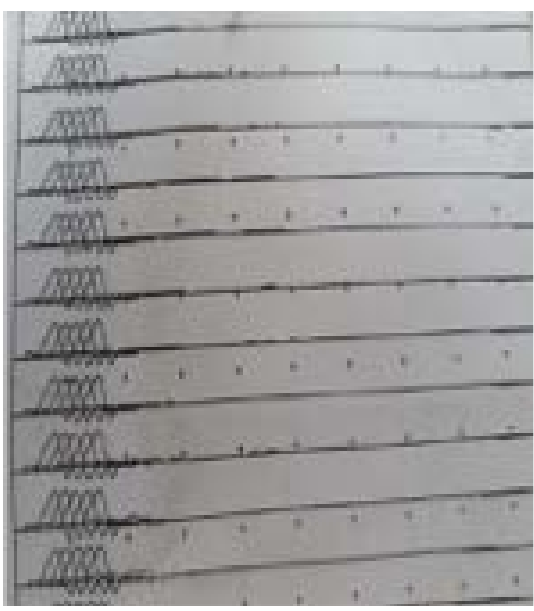

(a)

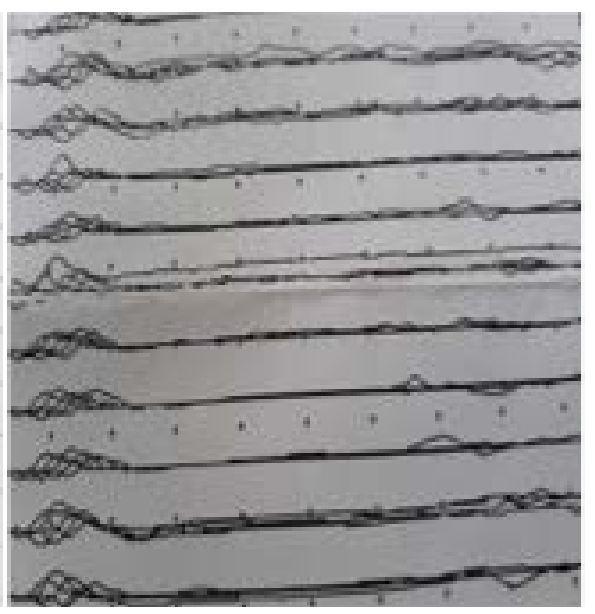

(b)

Figure 1. Results compatible with myasthenia gravis. (a) Large deltoid; (b) Orbicularis of the eyelid. 
who presented a peripheral neurogenic syndrome of 4 limbs and facial diplegia in December 2014 without sphincteric and respiratory disorders . Diffuse paresthesia was noted, especially in the lower limbs, two weeks before the clinical presentation. Lumbar puncture returned a clear fluid with a cytology less than one element, an albumin level of $2.26 \mathrm{~g} / \mathrm{L}$ and glucurachia of $0.60 \mathrm{~g} / \mathrm{L}$. The culture was negative. The electromyoneurogram (EMG) showed an axon-myelinic peripheral sensitive motor process of the 4 limbs with spot and segmental conduction blocks. The diagnosis of polyradiculoneuropathy on Sjörgen's type of connective tissue disease was chosen because of dry occulo-oral syndrome. antibodies against thymic cell extracts showed Anti-Sjögren's-syndrome-related antigen A, also called anti-Ro (SSA/Ro).

And Anti-Sjögren's-syndrome-related antigen A, also called anti-La (SSB/La) greater than 8 for the highest normal limit at 1.2. Biopsy of accessory salivary glands showed chronic grade IV sialitis of Chilchom and Masson. Six months later, the patient presented a more definite ptosis at night and an abnormal fatigability to the effort evoking a myasthenia associated. EMG confirms the myasthenic pattern and anti-acetylcholine receptor antibodies were positive (Figure 2).

The evolution was marked by a progressive recovery of motor skills and complete recovery at 6 months at the 4 limb level, but persistence of a more pronounced facial diparesis on the left and the regression of fatigability under medical treatment.

Myasthenia gravis was generally stable under corticotherapy and azathioprine, the management of the syndrome of sjogren was more difficult with many related complications.

\section{Observation 3}

This is a 20-year-old nulligravid patient followed for Graves' disease diagnosed in June 2010 and put on Carbimazole: 30 mg/day combined with Propanolol: $20 \mathrm{mg} /$ day.

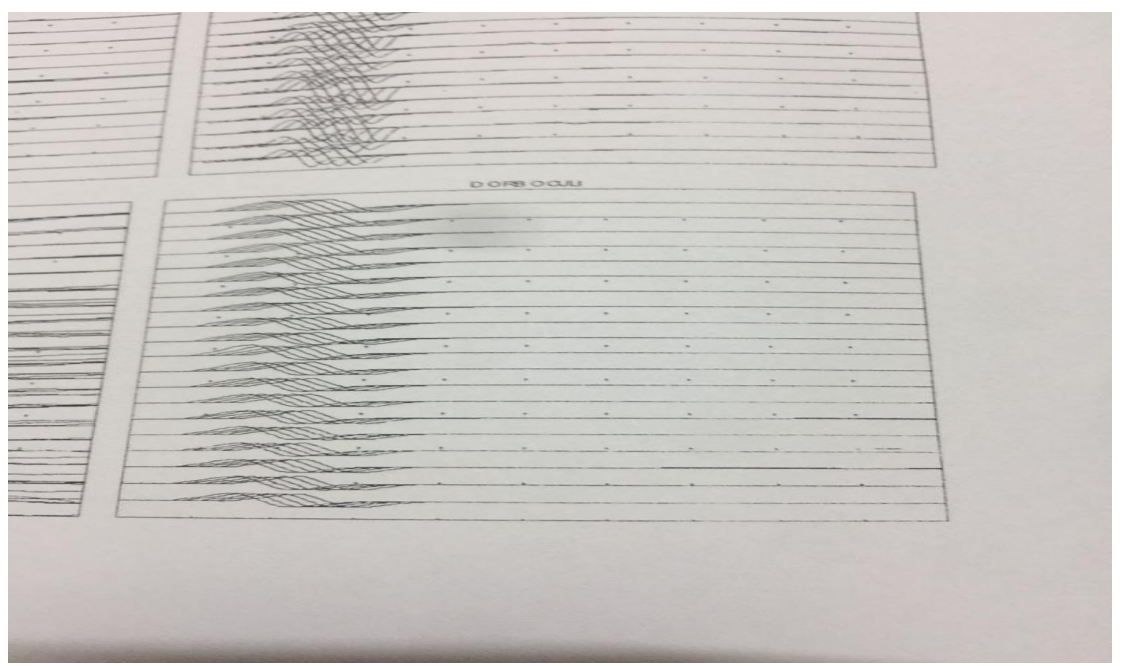

Figure 2. Decrease in neuromuscular conduction in orbicularis of the eyelid. 
She has presented since January 2012 fatigability to the effort. Later, appeared visual disorders (bilateral ptosis, intermittent diplopia) and an impossibility to completely chew solid food. The clinical picture was enriched in April 2012 with a nasal voice, a bilateral ptosis, a myogenic syndrome with fatigability to the effort. Approximately two hours after admission, there was acute respiratory failure with pharyngeal pain and respiratory congestion followed by reversible cardiorespiratory arrest after 5 minutes of external cardiac massage, Ambu mask ventilation, and Prostigmine test.

The explorations carried out on this occasion led to a progressive resumption of hyperthyroidism with THSus collapsed $<0.005$ (N: $0.1-4 \mathrm{mUI} / \mathrm{l})$ and T4l raised to 183 (N: 7 - $23 \mathrm{ng} / \mathrm{l}$ ). A brain MRI did not detect any abnormalities. The antinuclear antibody test returned positive to $1 / 160$ of homogeneous type as well as the antiU1 antibodies nuclear riboproteins positive to 9 .

The research of native anti-DNA Ab and anti-Sm Ab are negative.

Acetylcholine acetyl receptor antagonists were positive at $16.9 \mathrm{nmol} / \mathrm{l}(\mathrm{N}<$ $0.2)$ as were anti-TSH receptors: $2.4 \mathrm{IU} / \mathrm{l}(\mathrm{N}<1 \mathrm{IU} / \mathrm{l})$.

The electroneuromyogram at day 3 of hospitalization under prostigmine was in favor of progressive and severe generalized myasthenia gravis (Figure 3 ).

The thoracic CT found a small thymic remainder of $15 \mathrm{~mm}$.

The diagnosis of Graves' disease associated with myasthenia was discussed.

The evolution is marked by a regression of myasthenic signs with improvement of fatigability and masticatory disorders under medical treatment.

\section{Observation 4}

A 32-year-old patient had presented since April 2013 muscle weakness associated with abnormal fatigability to the effort with a worsening progression until 2015 when she got hospitalized. The interrogation found an effort fatigability sometimes associated with disorders of chewing and swallowing. The examination revealed: a myogenic syndrome with a muscular deficit, a left unilateral ptosis, a polyarthalgia of inflammatory appearance interesting the knees, the shoulders and the ankles, bilateral, symmetrical, chronic, not deforming, an oculo-oral dry syndrome.

Anti acetylcholine receptor $\mathrm{Ab}$ was positive at $24.8 \mathrm{nmol}$ (Normal < 0.40 ). Anti Ssa and SSb Ab were positive and salivary gland biopsy recovered to Chisholm stage III. The electromyogram showed a switchboard compatible with myasthenia (Figure 4).

Thoracic CT did not find a thymoma. The diagnosis of myasthenia gravis associated with Sjogren syndrome has been made.

Myasthenia gravis was generally stable under corticotherapy and azathioprine.

\section{Discussion}

Myasthenia gravis is an autoimmune disorder of the neuromuscular junction that can be associated with other autoimmune diseases. The diagnosis of all 4 observations was based on international consensus diagnostic criteria [3] [4] 


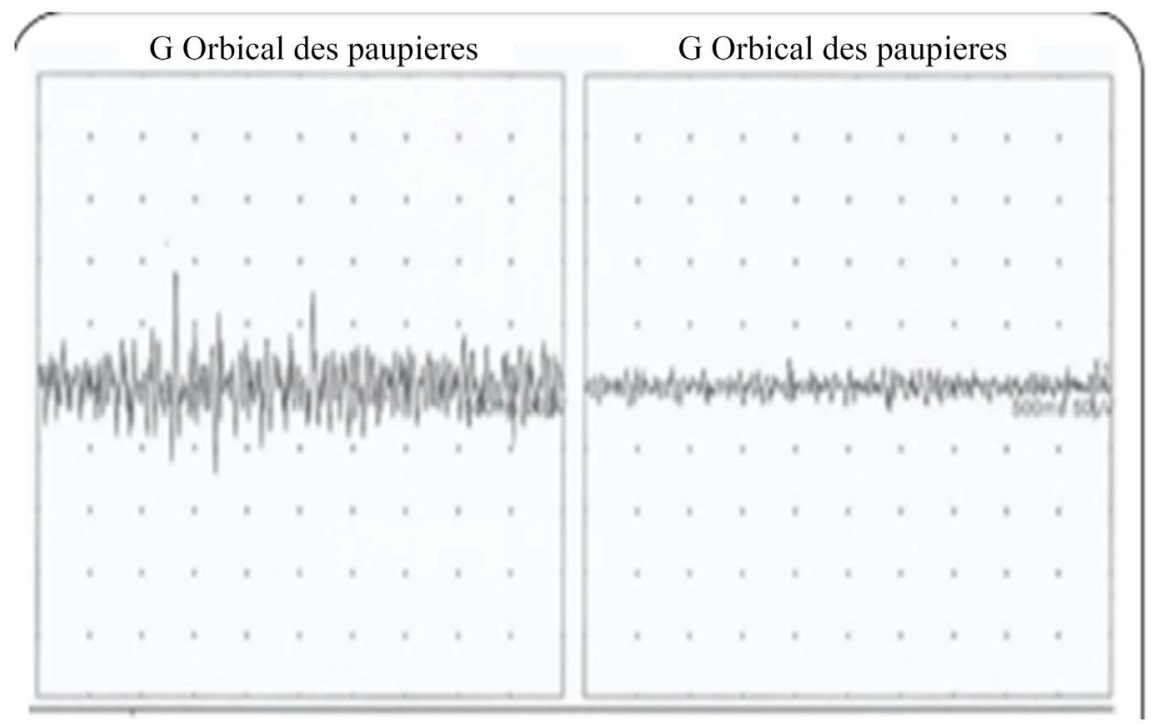

Figure 3. Decrease in neuromuscular conduction in orbicularis oculi.

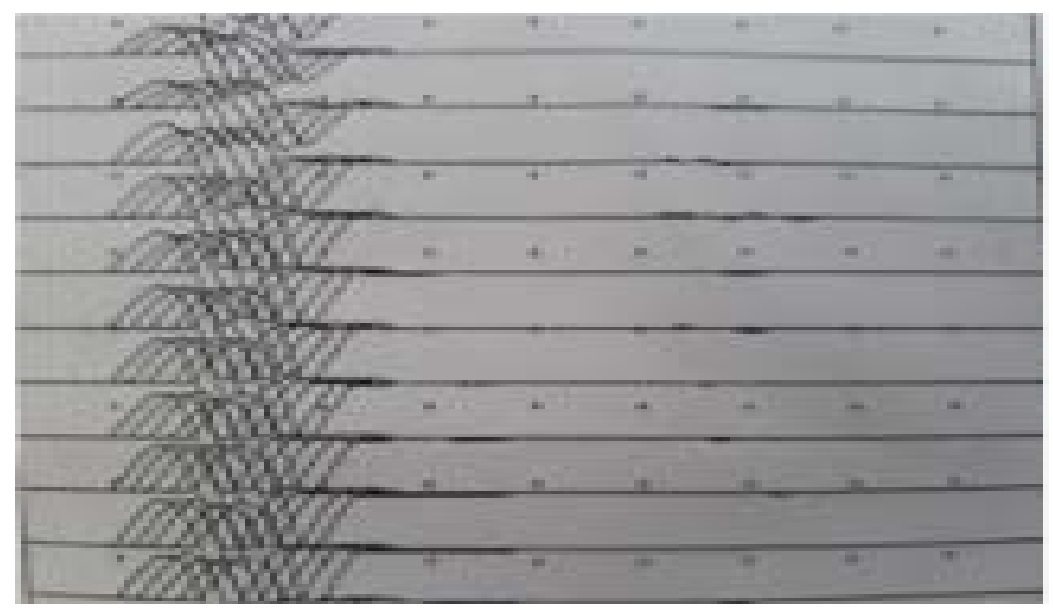

Figure 4. results compatible with myasthenia gravis; short abductor of the thumb.

[5]. There is a female predominance of these affections and this tendency is found with these patients that are all female. All autoimmune diseases can be associated with Myasthenia. Dysthyroidism remains the most commonly diagnosed condition with $5 \%$ to $10 \%$ of patients [2] and dysthyroidism is found in 3 of our 4 patients. With regard to autoimmune diseases described (Graves' disease, Biermer's disease, Sjogren's syndrome), their association with myasthenia remains rarely described [2]. Dysthyroidism remains the most common condition and its frequency is higher in myasthenic patients than in the general population [6]. The association myasthenia and Biermer's disease remains very poorly described in the literature. There are however rare cases of isolated associations and in the series of Goulon et al. [6], as Fraissse et al. [7], there were proportions respectively of $3 / 84$ patients who developed intrinsic anti-factor antibodies without any patient having developed an authentic Biermer's disease and 2/45. The association with Sjogren's syndrome, however, remains rarely described 
with 3 cases found in the literature; Indeed Dourov et al. [8] in France had described myasthenia gravis-associated with a thymoma and a malignant thymoma in 1968, in 2008 Sellami et al. [9] described another case associated with spondyloarthritis and a Gougerot and a third case was described by Tsai [10] associating a thymoma myasthenia and Sjogren syndrome. In our series of cases, we report a greater number of associations with sjogren syndrome, which is much more rarely described in the literature.

Myasthenia gravis illustrates the involvement of the thymus in autoimmune diseases because of its frequent association with thymic abnormalities and the important role of the thymus in its pathogenesis [11] even though in our series no patient presented a thymoma. The therapeutic strategies related to myasthenia were respected in the 4 patients [2]. All of our patients received prostigmine with intolerance in one patient, corticosteroid therapy was also instituted in all patients with definitive discontinuation in 2 patients. Azathioprine was initiated in 2 of our patients and weaning with corticosteroids was possible in these 2 patients despite the associated dysimmunity. High dose corticosteroids may achieve remission of both pathologies. In our cases, we started with a low dose of steroids, because a transitory worsening of MG that is sometimes observed on initiation of corticosteroids would have carried significant risk.

\section{Conclusion}

The association myasthenia and other diseases are not exceptional but are still poorly described in the literature. Dysthyroidism remains the most frequent in our series and in rare and isolated series in the literature. The search for other autoimmune diseases will depend on the clinical context even if the search for autoimmune dysthyroidism and thymoma should be systematic because of their frequent association with myasthenia.

\section{Conflicts of Interest}

The authors declare no conflicts of interest regarding the publication of this paper.

\section{References}

[1] Somnier, F.E. (2005) Increasing Incidence of Late-Onset Anti-AChR Antibody-Seropositive Myasthenia Gravis. Neurology, 65, 928-930. https://doi.org/10.1212/01.wnl.0000176067.32186.a3

[2] Eymard, B. (2014) Myasthenia Gravis on the Side of the Internist. The Journal of Internal Medicine, 35, 421-429. https://doi.org/10.1016/j.revmed.2013.08.013

[3] Evoli, A., Batocchi, A.P., Lo Monaco, M., et al. (1996) Clinical Heterogeneity of Seronegative Myasthenia Gravis. Neuromuscular Disorders, 6, 155-161.

https://doi.org/10.1016/0960-8966(96)00009-0

[4] Oosterhuis, H.J.G.H. (1984) Myasthenia Gravis. In: Clinical Neurology and Neurosurgery Monographs. Churchill Livingstone, Vol. 5.

[5] Rassmussen, A., et al. (2014) Comparison of the American-European Consensus 
Group Sjogren's Syndrome Classification Criteria to newly proposed American College of Rheumatology in a Large, Carefully Characterized Sicca Cohort. Annals of the Rheumatic Diseases, 73, 31-38

https://doi.org/10.1136/annrheumdis-2013-203845

[6] Trabelsi, L., Charfi, N., Triki, C., Mnif, M., Rekik, N., Mhir, C. and Abid, M. (2006) Myasthenia and Hyperthyroidism: Two Observations. Annales d Endocrinologie, 67, 265-269. https://doi.org/10.1016/S0003-4266(06)72597-5

[7] Goulon, M., Zittoun, J., Tuliez, M. and Estournet, B. (1979) Myasthenia and Pernicious Anemia. Neurology Review, 135, 605-614.

[8] Fraisse, T., Labauge, P., Comu, W., Arlaud, P. and De Waziere, B. (2007) Myasthenia of the Elderly: Diagnosis, Comorbidities and Evolution. La Presse Médicale, 36, 9-14. https://doi.org/10.1016/j.lpm.2006.07.003

[9] Sellami, M., Akrut, R., Feki, I., Ezzeddine, M., Hdiji, N., Mhiri, C. and Baklouti, S. (2008) Myasthenia-Spondylarthropathy: Fortuitous Association? About One Case and Review of the Literature. $A J N S, 27$.

[10] Tsai, Y., Lin, Y., Chen, C. and Tzao, C. (2013) Thymoma Associated with Myasthenia Gravis and Sjögren Syndrome. West Indian Medical Journal, 62, 264-265. https://doi.org/10.7727/wimj.2012.118

[11] Powerful, A. (2004) Thymic Function and Autoimmunity. The Journal of Internal Medicine, 25, 562-572. 